\title{
Penile Cancer pNX TNM Finding v8
}

National Cancer Institute

\section{Source}

National Cancer Institute. Penile Cancer pNX TNM Finding v8. NCI Thesaurus. Code C140070.

Penile cancer in which lymph node metastasis cannot be established. (from AJCC 8th Ed.) 